\title{
Src-family tyrosine kinase fyn phosphorylates phosphatidylinositol 3-kinase enhancer-activating Akt, preventing its apoptotic cleavage and promoting cell survival
}

\author{
$\mathrm{X}$ Tang $^{1}$, Y Feng ${ }^{2}$ and $\mathrm{K}$ Ye $\mathrm{e}^{\star, 1}$
}

Phosphatidylinositol 3-kinase enhancer-activating Akt (PIKE-A) binds Akt and upregulates its kinase activity, preventing apoptosis. PIKE-A can be potently phosphorylated on tyrosine residues 682 and 774 , leading to its resistance to caspase cleavage. However, the upstream tyrosine kinases responsible for PIKE-A phosphorylation and subsequent physiological significance remain unknown. Here, we show that PIKE-A can be cleaved by the active apoptosome at both D474 and D592 residues. Employing fyn-deficient mouse embryonic fibroblast cells and tissues, we demonstrate that fyn is essential for phosphorylating PIKE-A and protects it from apoptotic cleavage. Active but not kinase-dead fyn interacts with PIKE-A and phosphorylates it on both $\mathrm{Y} 682$ and $\mathrm{Y} 774$ residues. Tyrosine phosphorylation in PIKE-A is required for its association with active fyn but not for Akt. Mutation of $D$ into A in PIKE-A protects it from caspase cleavage and promotes cell survival. Thus, this finding provides a molecular mechanism accounting for the antiapoptotic action of src-family tyrosine kinase.

Cell Death and Differentiation (2007) 14, 368-377. doi:10.1038/sj.cdd.4402011; published online 14 July 2006

PIKE (phosphatidylinositol (PI) 3-kinase enhancer) was originally identified as a nuclear GTPase, which stimulates the lipid kinase activity of nuclear PI 3-kinase. ${ }^{1}$ PIKE interacts with numerous signaling proteins, including PI 3-kinase and PLC- $\gamma 1$ through its N-terminus. ${ }^{2}$ Accumulating evidence reveals that PIKE plays an important role in preventing neuronal apoptosis. ${ }^{3,4}$ Phosphatidylinositol 3-kinase enhancer-activating Akt (PIKE-A) is an isoform of PIKE gene, and primarily localizes in the cytoplasm. PIKE-A lacks the $\mathrm{N}$-terminal segment, where PI 3-kinase binds. Interestingly, PIKE-A directly binds to activated Akt but not PI 3-kinase in a guanine nucleotide-dependent way and stimulates the kinase activity of Akt. ${ }^{5,6}$ PIKE-A/Akt interaction is mediated through GTPase domain of PIKE-A and the C-terminal regulatory domain and a portion of catalytic domain of Akt. Overexpression of wild-type PIKE-A enhances Akt activity, whereas dominant-negative PIKE-A or PIKE-A knockdown inhibits it. PIKE-A is coamplified with CDK4 in a variety of human cancers. The overexpressed PIKE-A promotes cancer cell invasion and prevents apoptosis. ${ }^{5,6}$ Recently, we have shown that PIKE is potently phosphorylated on tyrosine residues, leading to its resistance against the proteolytic cleavage by the in vitro cell-free apoptosomes and in apoptotic cells. EGF triggers PIKE-A phosphorylation on both Y774 and Y682 residues. Diminishing tyrosine phosphorylation on PIKE-A by mutating either $Y 774$ or $Y 682$ into $F$ makes PIKE-A susceptible to apoptotic degradation. Moreover, growth factor pretreatment also attenuates the apoptotic cleavage of PIKE-A in human cancer cells. ${ }^{7}$ Thus, tyrosine phosphorylation may suppress proteolytic cleavage of PIKEA. However, the upstream tyrosine kinases responsible for this effect remain elusive.

The src family of nonreceptor protein tyrosine kinases, including src, lyn, fyn, Ick, hck, fgr, blk and yes, play an important role in cell cycle control, cell adhesion and movement, and cell proliferation and differentiation. ${ }^{8,9}$ All src family members are composed of several well-characterized functional domains. The amino-terminus of src kinases is myristoylated, which is essential for targeting of src kinases to the inner leaflet of cell membranes. Src also contains both $\mathrm{SH} 3$ and $\mathrm{SH} 2$ (src homology) domains, followed by the catalytic tyrosine kinase domain and by a region in the carboxyl-terminus that contains a conserved regulatory tyrosine phosphorylation site. ${ }^{8}$ Src kinases are activated by a variety of cell surface receptors, G-protein-coupled receptors ${ }^{10}$ and by growth factor receptor stimulation. ${ }^{11}$ Fyn, a critical member of src family tyrosine kinase, mediates a variety of cellular processes, including signaling via the T-cell receptor, ${ }^{12}$ regulation of brain function ${ }^{13,14}$ as well as adhesion-mediated signaling. ${ }^{15,16}$ It is worth noting that src tyrosine kinases including fyn also mediate cell survival. For instance, expression of $\mathrm{v}$-src can rescue certain cells from apoptosis caused by cytokine deprivation or disruption of cell binding to the extracellular matrix. ${ }^{17}$ Detachment of intestineal

\footnotetext{
${ }^{1}$ Department of Pathology and Laboratory Medicine, Emory University School of Medicine, Room 145, Whitehead Building, 615 Michael Street, Atlanta, GA 30322 , USA and ${ }^{2}$ Department of Pharmacology, Emory University School of Medicine, Atlanta, GA 30322, USA

${ }^{*}$ Corresponding author: K Ye, Department of Pathology and Laboratory Medicine, Emory University School of Medicine, Room 145, Whitehead Building, 615 Michael Street, Atlanta, GA 30322, USA. Tel: + 404712 2814; Fax: + 404712 2979; E-mail: kye@emory.edu

Keywords: src tyrosine kinase; fyn; PIKE-A; apoptotic cleavage; cell survival

Abbreviations: PIKE, phosphatidylinositol 3-kinase enhancer; PIKE-A, PIKE-activating Akt; PZA domains, PH, ArfGAP (zinc-finger) and ankyrin repeats domains; FAK, focal adhesion kinase; pyk2, proline-rich tyrosiney kinase 2

Received 20.4.06; revised 08.6.06; accepted 09.6.06; Edited by M Blagosklonny; published online 14.7.06
} 
epithelial cells triggers a transient c-src and c-fyn activation, antagonizing detachment-induced anoikis in these cells. ${ }^{18}$ Loss of Ick has no effect on the survival of peripheral T cells; however, deletion of both lck and fyn substantially diminishes its survival. ${ }^{19}$ TRANCE-induced survival of osteoclasts isolated from src -/- mice is compromised relative to wild-type mice. Similarly, TRANCE-induced activation of Akt is significantly reduced in src -/- osteoclasts compared to wild-type osteoclasts, suggesting that the protective signals from src family kinases are mediated by $\mathrm{PI}$ 3-kinase/Akt signaling. ${ }^{20}$

In this report, we show that active but not kinase-dead fyn binds PIKE-A and phosphorylates it on both Y682 and Y774 residues. Mutation of $Y 682$ and $Y 774$ into $F$ abolishes the interaction between PIKE-A and fyn. However, PIKE-A tyrosine phosphorylation is not required for Akt association. The C-terminal kinase domain of fyn potently binds to ArfGAP domain in PIKE-A. Moreover, active fyn but not src protects PIKE-A from apoptotic degradation, which occurs at D474 and D592 residues. Mutation of D into A in PIKE-A protects it from apoptotic cleavage and promotes cell survival.

\section{Results}

D474 and D592 residues are the major apoptotic cleavage sites in PIKE-A. PIKE-A can be fragmented into multiple segments during apoptosis. Previous study demonstrates that $\mathrm{PH}$ domain and ArfGAP domain possess the cleavage sites by active caspases. The putative caspase3 cutting motif DLDD634 is not the cleavage site. ${ }^{7}$ There are nine and 10 aspartate residues (D) in $\mathrm{PH}$ domain and ArfGAP domain, respectively. Based on the proteolytic fragment sizes from these two domains, we chose numerous potential cleavage positions and generated the point mutants with $D$ into $\mathrm{A}$ in PZA domains ( $\mathrm{PH}$, ArfGAP (zinc-finger) and ankyrin repeats domains) of PIKE-A, and transfected into HEK293 cells. In vitro cleavage assay with transfected cell lysate manifested that GFP-PZA was potently cleaved into a few segments, yielding a prominent band with molecular weight of $60 \mathrm{kDa}$ and two weak fragments at 50 and $45 \mathrm{kDa}$. D592A mutation in PZA substantially decreased two other cutting sites cleavage, indicating that D592 cleavage is prerequisite for the other two sites to be further processed. Interestingly, the minor 50 and $45 \mathrm{kDa}$ fragments were suppressed when D474 was mutated into $A$, although the $60 \mathrm{kDa}$ cleaved fragment was still detectable (Figure 1a). These results demonstrate that both D474 and D592 are the major cutting sites, with D592 being the initial proteolytic cleavage site, fitting with previous data that $\mathrm{PH}$ domain and ArfGAP domain each contains cutting sites. Presumably, GFP-PZA also contains a minor cutting site between 474 and 592 . Cleavage at D592 might initiate its apoptotic degradation, accounting for the production of $50 \mathrm{kDa}$ fragment.

a

b

myc-PIKE-A
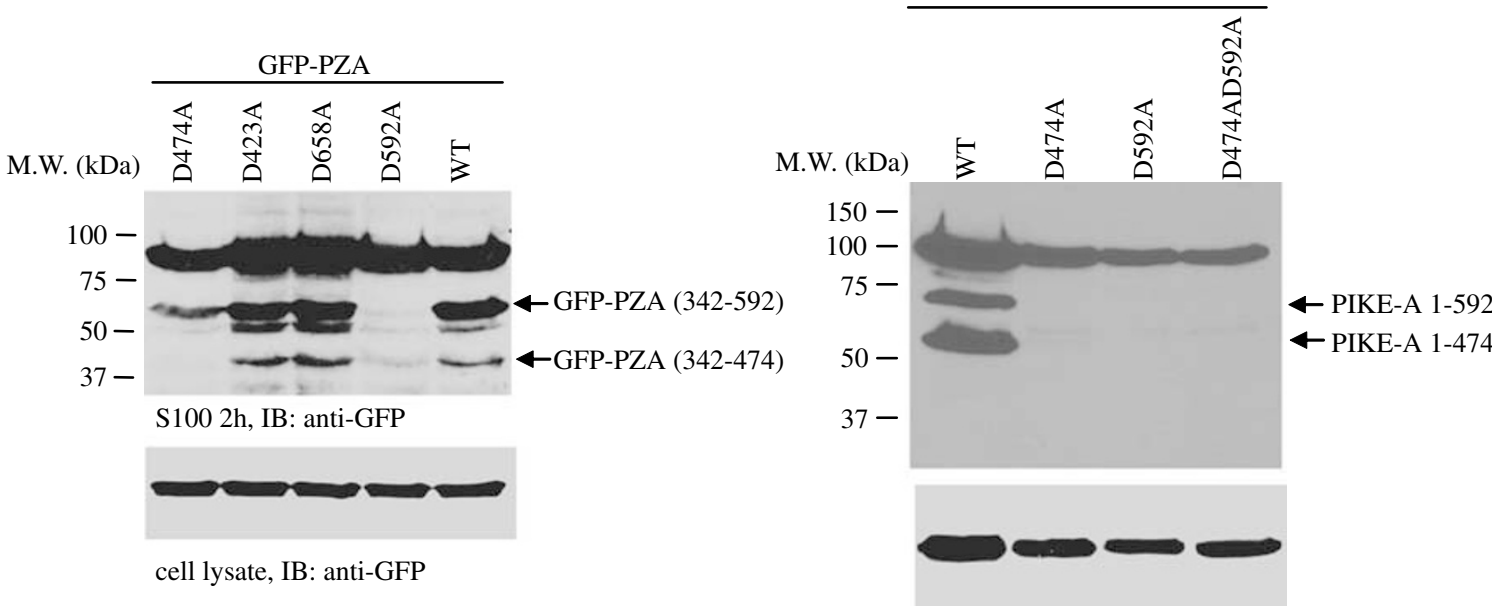

C

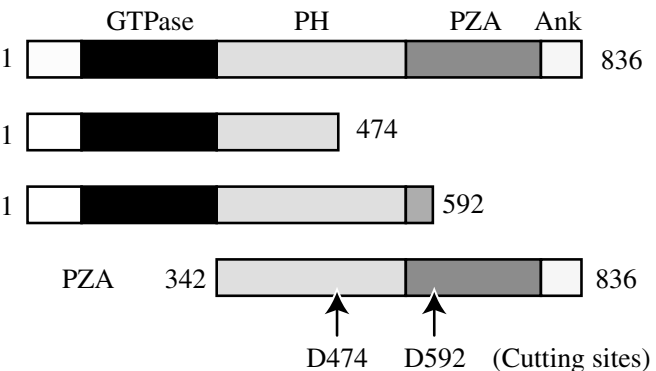

$92 \mathrm{kDa}$

$53 \mathrm{kDa}$

$65 \mathrm{kDa}$

$55 \mathrm{kDa}$

Figure 1 D474 and D592 residues are the apoptotic cleavage sites in PIKE-A. (a) In vitro PIKE-A fragment apoptotic cleavage assay. Wild-type PIKE-A fragment yielded a prominent product with $60 \mathrm{kDa}$ and two minor bands at 50 and $45 \mathrm{kDa}$. D592A mutation blocked PZA fragment proteolytic degradation. D474A abolished 50 and $45 \mathrm{kDa}$ protein production, but the $60 \mathrm{kDa}$ band was still detectable. (b) D474,592A mutation prevent PIKE-A apoptotic degradation. After staurosporine treatment, wild-type PIKE-A was profoundly cleaved, generating a couple of fragments with molecular weight at about 65 and $53 \mathrm{kDa}$, which correspond to 1-592 and 1-474 fragments, respectively. PIKEA degradation was markedly blocked in all D474A, D592A and (D474,592A) PIKE-A mutants. (c) Diagram of PIKE-A and its proteolytic fragments 
To further evaluate whether both sites are responsible for full-length PIKE-A degradation, we prepared Myc-PIKE-A constructs with either D474 or D592 or both residues mutated into alanine. PIKE-A cleavage was markedly inhibited in the single-point mutants and completely blocked when both D474 and D592 were mutated into A (Figure 1b), suggesting that both D474 and D592 sites are two major proteolytic cleavage sites on PIKE-A. Blockage of one cutting site profoundly circumvents another position cleavage in full-length PIKE-A.

\section{Active fyn tyrosine kinase binds PIKE-A and phospho-} rylates it. Previous study reveals that Y682 and Y774 residues in PIKE-A are potently phosphorylated upon EGF stimulation, which can be completely blocked by EGF receptor inhibitor AG1296. However, active EGF receptor tyrosine kinase cannot directly phosphorylate PIKE-A, ${ }^{7}$ indicating that the downstream cytoplasmic tyrosine kinases might be implicated in this event. To search for the tyrosine kinases responsible for the effect, we examined a variety of cytoplasmic tyrosine kinases including focal adhesion kinase (FAK), src, fyn, etc. We co-transfected GST-PIKE-A into HEK293 cells with various HA-tagged tyrosine kinases, and pulled down PIKE-A with glutathione beads. Its tyrosine phosphorylation status was monitored with phosphotyrosine-specific anti-PY99 antibody. PIKE-A was robustly phosphorylated by active fynA (Y528F), and it was also phosphorylated by active srcA (Y527F). By contrast, no phosphorylation was detected in FAK-, wild-type src-, kinasedead src- (K295R) or fynD- (K296R) transfected samples (Figure 2a, top panel). Interestingly, we noticed a $60 \mathrm{kDa}$ tyrosine-phosphorylated protein co-precipitated with PIKE-A in both active fynA and srcA samples, resembling tyrosine phosphorylated src and fyn. Immunoblotting with anti-HA antibody verified that active fyn strongly bound to PIKE-A, and active src weakly associated with PIKE-A (Figure 2a, second panel). The expression of transfected constructs was confirmed (Figure 2a, third and bottom panels). To investigate whether src and fyn directly phosphorylate PIKE-A, we performed an in vitro kinase assay with purified wild-type and mutant GST-PIKE-A proteins using active src and fyn. Wild-type PIKE-A was evidently phosphorylated by both kinases, mutation of either Y682 or Y774 into F substantially diminished its phosphorylation. No phosphorylation was detected when both $\mathrm{Y} 682$ and $\mathrm{Y} 774$ residues were mutated in PIKE-A (Figure 2b). To further assess PIKE-A tyrosine phosphorylation by src and fyn in intact cells, we cotransfected active src and fyn into HEK293 cells with wildtype and mutant GST-PIKE-A, respectively. As expected, wild-type PIKE-A was robustly phosphorylated by active fyn. Mutation of either $Y 682$ or $Y 774$ into $F$ significantly decreased PIKE-A phosphorylation. No phosphorylation was detectable when both residues were mutated, indicating that Y682 and Y774 residues are selectively phosphorylated by fyn in cells. Surprisingly, both wild-type PIKE-A and its mutants were potently phosphorylated by active src, suggesting that other tyrosine residues in PIKE-A can also be phosphorylated by active src itself or its downstream tyrosine kinases in intact cells (Figure 2c, top panel). Active fyn and src specifically interacted with wild-type but not mutated PIKE-A, demonstrating that phosphorylation of both
Y682 and $Y 774$ residues is required for active fyn and src to associate with PIKE-A (Figure 2c, second panel). To examine whether tyrosine phosphorylation in PIKE-A also regulates its interaction with active Akt, we co-transfected HEK293 cells with Akt, and stimulated cells with EGF. GSTpull down assay revealed that Akt robustly bound to both wild-type and mutated PIKE-A (Figure 2d, top panel), demonstrating that tyrosine phosphorylation is unnecessary for PIKE-A binding to Akt. Taken together, these findings demonstrate that tyrosine phosphorylation on PIKE-A is essential for its association with fyn and src, but it does not implicate in the association between PIKE-A and Akt.

Tyrosine kinase domain in fyn binds to ArfGAP domain in PIKE-A. To map which portion of PIKE-A binds to fyn, we co-transfected active HA-fynA into HEK293 cells with various myc-tagged PIKE-A fragments. Fyn was immunoprecipitated and the co-precipitated proteins were analyzed with anti-myc antibody. Full-length PIKE-A bound to active fyn. Deletion of the N-terminal 1-72 residues or GTPase fragment enhanced the association between PIKE-A and active fyn. The strongest binding occurred to PZA domain fragment, when the N-terminal 1-356 residues were eliminated. Interestingly, the N-terminal 1-365 segments also faintly associated with active fynA (Figure 2a, top panel). These data indicate that C-terminus of PIKE-A is essential for its binding to active fynA. To determine the exact motif in PIKE-A responsible for binding to active fynA, we performed in vitro binding assay with cell lysate from HEK293, which was transfected with active fynA. Truncation assay demonstrated that ArfGAP domain, where Y682 resides, is critical for PIKE-A to interact with fyn (Figure 3b, top panel).

To examine which domain in fyn interacts with PIKE-A, we purified a variety of GST-fyn fragments, and incubated with lysate of HEK293 cells, which were transfected with mycPIKE-A and treated with or without EGF for $10 \mathrm{~min}$. Strongest binding by PIKE-A occurred to the C-terminal $\mathrm{SH} 1$ domain (kinase domain). The N-terminal 1-250 segments, which contains $\mathrm{SH} 4, \mathrm{SH} 3$ and $\mathrm{SH} 2$ domains, also faintly interacted with PIKE-A. However, SH3 domain alone barely associated with PIKE-A, regardless of EGF stimulation (Figure $3 \mathrm{c}$, top panel). Co-precipitation assay revealed that active fynA but not kinase-dead fynD specifically interacted with wild-type PIKE-A. None of the PIKE-A mutants associated with either active or kinase-dead fyn (Figure 3d). Therefore, fyn kinase activity is necessary for its association with PIKE-A, and tyrosine phosphorylation on PIKE-A is also indispensable for the interaction. To further explore the notion that tyrosine phosphorylation on PIKE-A is required for its association with active fyn, we transfected HEK293 cells with GST-PIKE-A, and pretreated the transfected cells with various tyrosine kinase inhibitors, followed by EGF stimulation. GST pull-down assay revealed that EGF treatment enhanced fyn binding to PIKE-A, compared with control. Tyrosine kinase inhibitors, except AG490, markedly blocked the interaction (Figure 3e, top panel). Immunoblotting analysis showed that PIKE-A was tyrosine phosphorylated when the interaction occurred, underscoring that PIKE-A tyrosine phosphorylation is essential for the complex formation (Figure $3 e$, second panel). Taken together, these findings demonstrate that ArfGAP 
a

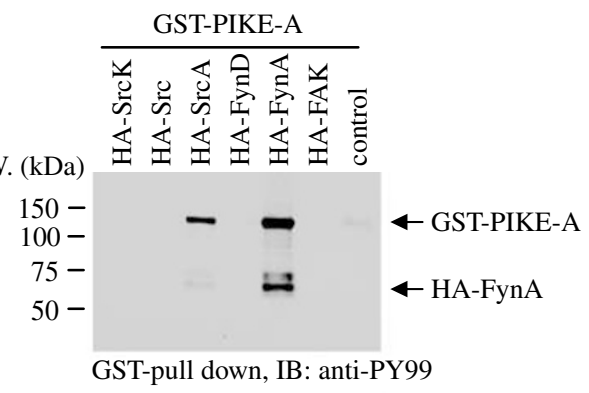

M.W. (kDa)

GST-pull down, IB: anti-PY99

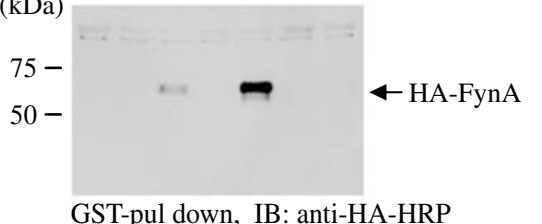

GST-pul down, IB: anti-HA-HRP

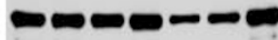

cell lysate, IB: anti-HA-HRP

M.W. (kDa)

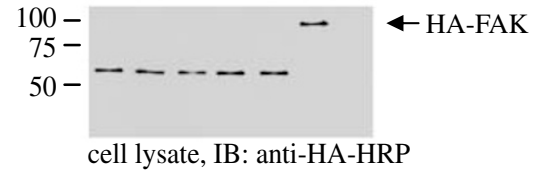

b
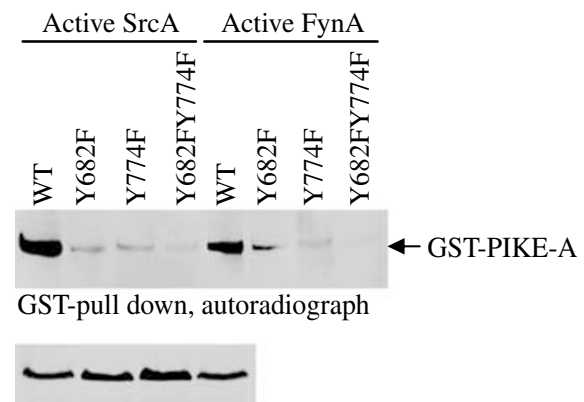

cell lysate, IB: anti-GST-HRP

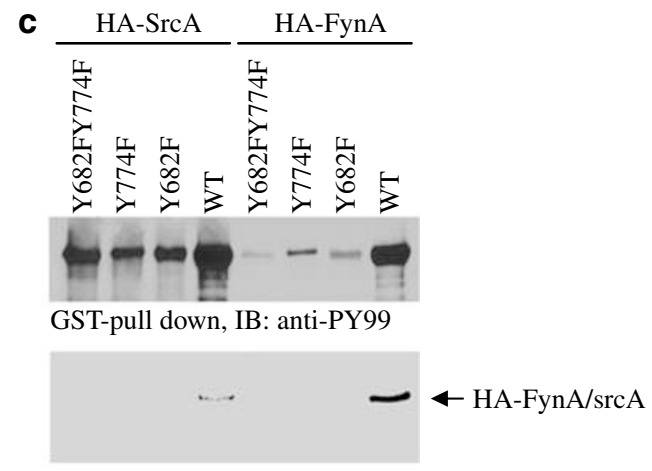

cell lysate, IB: anti-HA-HRP
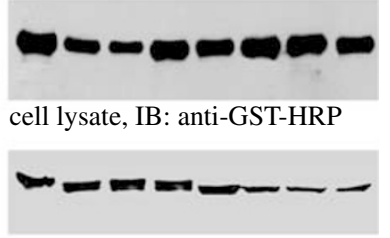

cell lysate, IB: anti-HA-HRP d

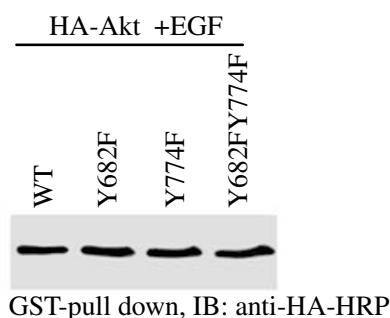

cell lysate, IB: anti-GST-HRP

cell lysate, IB: anti-HA-HRP

Figure 2 Active fyn tyrosine kinase binds PIKE-A and phosphorylates it. (a) Active fyn and src tyrosine kinases phosphorylate PIKE-A in cells. By contrast, no phosphorylation was detected by FAK, wild-type or kinase-dead src or fyn (top panel). Only active but not kinase-dead fyn or src selectively interacted with PIKE-A, but fynA revealed much stronger binding affinity than srCA (second panel). The expression of transfected GST-PIKE-A and HA-tagged tyrosine kinases were verified by immunoblotting analysis (third and bottom panels). (b) Active src and fyn kinases can phosphorylate PIKE-A in vitro. Wild-type PIKE-A was strongly phosphorylated by both kinases; however, the phosphorylation was substantially decreased in PIKE-A mutants (upper panel). Equal amount of PIKE-A was employed (lower panel). (c) Active fyn and src distinctively phosphorylate wild-type and mutated PIKE-A in cells. Active fynA selectively phosphorylated wild-type PIKE-A, but active src phosphorylated both wild-type and mutant PIKEA in transfected cells (top panel). Only wild-type PIKE-A selectively bound to active fynA and srcA. However, fynA bound to PIKE-A much stronger than srcA (second panel). The expression of transfected PIKE-A and tyrosine kinase plasmids was confirmed (third and bottom panels). (d) Akt bound to both wild-type and tyrosine-mutated PIKE-A (top panel). The expression of Akt and PIKE-A constructs was verified (middle and bottom panels)

domain in PIKE-A is implicated in binding to active fyn through its kinase domain.

Tyrosine phosphorylation prevents PIKE-A apoptotic cleavage. Mutation of either $\mathrm{Y} 682$ or $\mathrm{Y} 774$ into $F$ incurs PIKE-A vulnerable to active apoptosome-mediated degradation, and EGF treatment alleviates PIKE-A cleavage in vitro and in vivo. ${ }^{7}$ To define the role of tyrosine phosphorylation by active src and fyn in preventing PIKE-A apoptotic fragmentation, we co-transfected active srcA and fynA into HEK293 cells with wild-type and mutated GST-PIKE-A, respectively. PIKE-A was pulled down, and incubated in the cell-free apoptotic solution. The strongest degradation occurred in samples when both $\mathrm{Y} 682$ and $\mathrm{Y} 774$ residues were mutated into $F$, followed by single mutants, whereas wild-type PIKE-A remained intact. Compared to active fynA, 
a
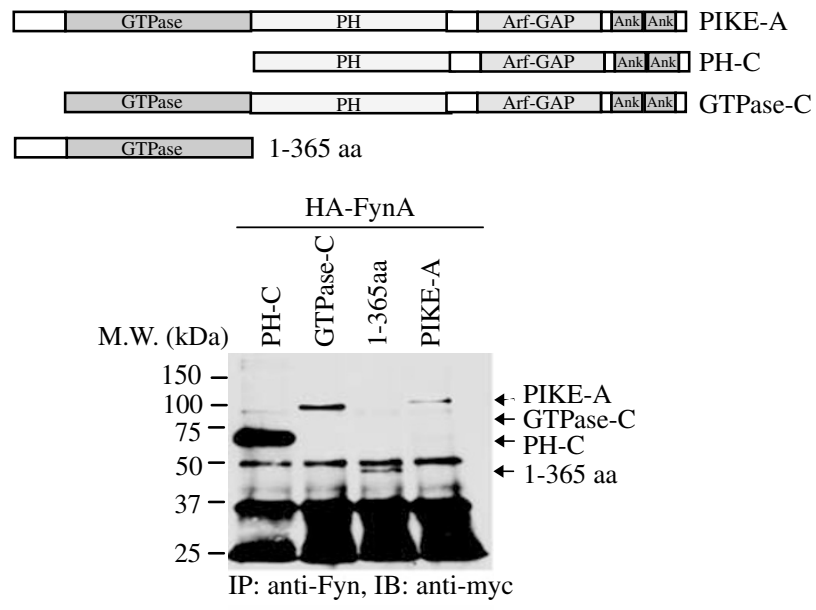

M.W. (kDa)

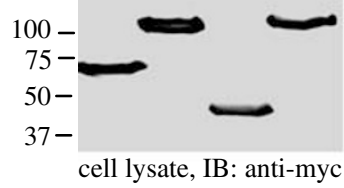

cell lysate, IB: anti-HA-HRP

C
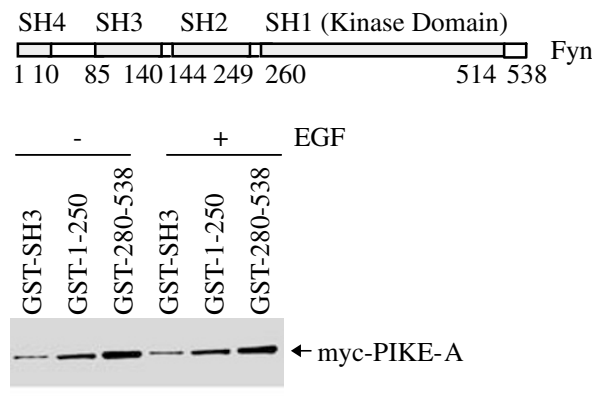

GST-pull down, IB: anti-myc

M.W. (kDa)

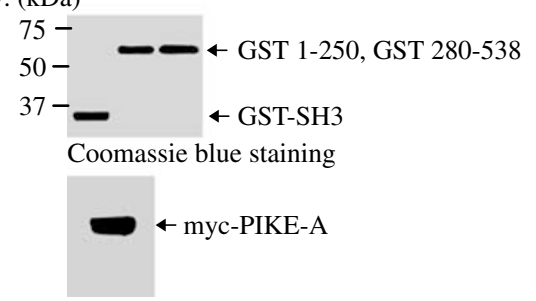

cell lysate, IB: anti-myc b

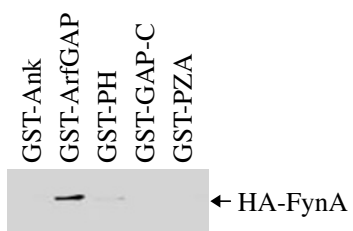

M.W. (kDa)

GST-pull down, IB: anti-Fyn

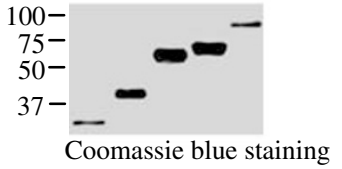

$\leftarrow$ HA-FynA

cell lysate, IB: anti-HA-HRP

d

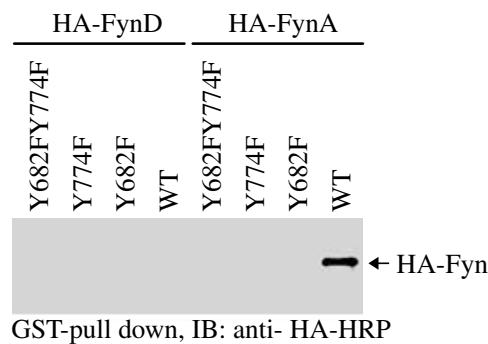

0000

cell lysate, IB: anti-HA-HRP

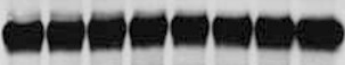

cell lysate, IB: anti-GST-HRP

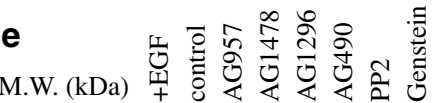

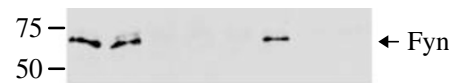

GST-pull down, IB: anti-Fyn

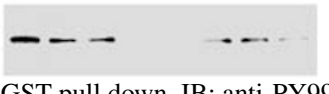

GST-pull down, IB: anti-PY99

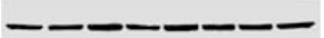

cell lysate, IB: anti-GST-HRP

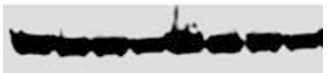

cell lysate, IB: anti-Fyn

Figure 3 Tyrosine kinase domain in fyn binds to ArfGAP domain in PIKE-A. (a) Mapping of PIKE-A binding domain for active fyn kinase. The strongest interaction occurred to N-terminal GTPase-truncated fragment (lane 1), followed by N-terminal 1-72 residues deleted segment (GTPase-C). The weak binding was also observed on full-length PIKE-A. The N-terminal 1-356 residues faintly bound to fynA as well (top panel). The expression of transfected PIKE-A fragments and fyn was confirmed (middle and bottom panels). (b) ArfGAP domain in PIKE-A is required for its association with fynA. ArfGAP domain displayed strongest interaction with active fynA (upper panel). The purity of GST fusion proteins was verified with Coomassie blue staining (middle panel). (c) The catalytic domain in fyn strongly binds to PIKE-A. The C-terminal kinase domain in fyn revealed the strongest binding affinity to PIKE-A, which was followed by the N-terminal fragment. By contrast, SH3 domain alone did not bind to PIKE-A (top panel). The purity of GSTfyn fragment proteins was confirmed by Coomassie blue staining. (d) Fyn kinase activity is required for its association with PIKE-A. Active but not kinase-dead fyn selectively bound to wild-type PIKE-A (top panel). (e) Tyrosine kinase inhibitors disrupt the interaction between PIKE-A and fyn. PIKE-A bound fyn under controlled condition, and EGF enhanced the association. Tyrosine kinase inhibitors, which abolished PIKE-A phosphorylation, blocked the interaction between PIKE-A and fyn (top and second panels). The expression of transfected PIKE-A and endogenous fyn kinase was confirmed (third and bottom panels) 
the mutant samples from active srcA transfection revealed stronger digestion, although wild-type PIKE-A was relatively resistant somehow (Figure 4a). To further explore the physiological role of src and fyn in preventing PIKE-A apoptotic degradation, we employed src- or fyn-deficient mouse embryonic fibroblast (MEF) cells. We treated wildtype and knockout cells with staurosporine in the presence or absence of EGF, and monitored PIKE-A cleavage by immunoblotting with anti-PIKE-A antibody, raised against the $\mathrm{N}$-terminal 1-72 residues. Compared with wild-type cells, PIKE-A was almost completely degraded in fyn knockout cells, which was partially digested in src-null cells, regardless of EGF treatment (Figure 4b, top panel). PARP cleavage pattern tightly correlated with PIKE-A apoptotic degradation (second panel). EGF provoked potent PIKE-A tyrosine phosphorylation in wild type, which was moderately decreased in src - / - cells and completely abolished in fyn $-/$ - cells (third panel), supporting that fyn is critical for phosphorylating PIKE-A in cells.

To further evaluate the physiological role of fyn in mediating PIKE-A tyrosine phosphorylation and apoptotic degradation, we conducted PIKE-A proteolytic cleavage assay with fyndeficient mouse tissues. PIKE-A was immunoprecipitated from wild-type and fyn-null mouse brain and liver, followed by incubation in the cell-free apoptotic solution. Compared with PIKE-A from wild-type mouse, PIKE-A from fyn -/- tissues was markedly cleaved (Figure 4c, left top panel). Similar levels of PIKE-A were immunoprecipitated from same tissue in both $+/+$ and $-I-$ mice (left second panel). In alignment with this observation, PIKE-A phosphorylation was profoundly decreased in fyn $-/-$ mouse (left third panel). Moreover, PIKE-A tyrosine phosphorylation in brain was much stronger than in the liver, fitting with higher expression level of PIKE-A in the brain than in the liver. Fyn depletion in knockout mouse was verified (right top panel). Interestingly, phosphorylation of GSK-3 was inhibited in knockout mouse (right second panel), suggesting that disruption of fyn affects the activity of Akt presumably through PIKE-A. Therefore, fyn plays an essential role for protecting PIKE-A from apoptotic cleavage, and src might also contribute to this effect as well.

Caspase-resistant PIKE-A mutants promotes cell survival. PIKE-A promotes cell survival through upregulating Akt, a crucial serine/threonine kinase for antagonizing apoptosis 5 and 6 . To examine the biological effect of caspase-resistant PIKE-A mutants on cell survival, we transfected HeLa cells with wild-type PIKE-A, D474A, D592A and D474,592A constructs, and treated the transfected cells with staurosporine. Compared with control, overexpression of caspase-resistant PIKE-A mutants decreased DNA fragmentation and caspase- 3 activity. The strongest inhibition was observed in PIKE-A- (D474, 592A) transfected cells, followed by D592A and D474A (Figure $5 a$ and b). Quantitative analysis revealed that more than $50 \%$ cells were in apoptosis in control cells. Transfection of wild-type PIKE-A slightly decreased programmed cell death. The apoptotic ratio was, respectively, decreased to $39.2,29$ and $26.8 \%$ in caspase-resistant PIKE-A construct-transfected cells (Figure $5 \mathrm{c}$ ). Therefore, prevention of PIKE-A from apoptotic degradation enhances its stimulatory effect on cell survival.

To determine the role of fyn-mediated PIKE-A tyrosine phosphorylation in antagonizing apoptosis, we co-transfected active fynA or srcA into HeLa cells with wild-type PIKE-A and mutants with $Y$ into $F$, respectively, and treated cells with staurosporine. When co-transfected with fynA, wild-type PIKE-A evidently diminished DNA fragmentation (Figure 5d, top panel). In contrast, evident DNA fragmentation occurred in Y682F-, Y774F- and Y682,774F-transfected cells. By contrast, co-transfection of srcA and wild-type or mutated PIKE-A barely exhibited any protective effect. Immunoblotting analysis revealed that PARP cleavage coupled to DNA fragmentation effect. As a control, equal amount of protein sample was loaded (Figure 5d, middle and lower panels). Collectively, our findings demonstrate that caspase-resistant PIKE-A mutants suppress apoptosis. Fyn phosphorylates PIKE-A and prevents its apoptotic degradation, leading to promotion of cell survival.

\section{Discussion}

In the present study, we have identified that fyn acts as a major physiological tyrosine kinase for PIKE-A. Tyrosine phosphorylation on PIKE-A in wild-type but fyn-null MEF cells or mice tightly correlates with its resistance against apoptotic degradation. Further, we have also shown that PIKE-A is cleaved at D474 and D592 sites during apoptosis. Mutation of D474 and D592 into A renders PIKE-A to resist against caspase-mediated cleavage, resulting in escalating cell survival. Although src might somewhat contribute to PIKE-A phosphorylation in vivo, PIKE-A enhances cell survival in the presence of active fyn but not src. Thus, fyn is implicated in PIKE-A phosphorylation and resistance to caspase-mediated cleavage, which might provide a molecular mechanism accounting for antiapoptotic action of fyn.

Y682 and Y774 are two main residues phosphorylated by fyn both in vitro and in transfected cells (Figure 2). In fyn-deficient cells, PIKE-A tyrosine phosphorylation was completely blocked (Figure 4), underscoring that fyn is the major tyrosine kinase phosphorylating PIKE-A in cells. Nevertheless, PIKE-A can also be phosphorylated by active src in vitro and in transfected cells. Mutation of either Y682 or Y774 residue abolishes PIKE-A tyrosine phosphorylation in vitro; however, transfection of active src still strongly phosphorylated the mutated PIKE-A in transfected cells (Figure $2 b$ ), suggesting that active src or its downstream kinases could phosphorylate other tyrosine residues in PIKE-A in transfected cells. In src-null cells, PIKE-A tyrosine phosphorylation is decreased but still detectable, indicating that src is not the major physiological kinase for PIKE-A. The complete loss of PIKE-A tyrosine phosphorylation in fyn-null cells demonstrated that fyn is the key upstream tyrosine kinase for PIKE-A in vivo.

Previous study demonstrates that PIKE-A interacts with the C-terminal regulatory and partial kinase domains of Akt through its GTPase domain. PIKE-A selectively binds active phosphorylated Akt. ${ }^{5,6}$ Consistent with these findings, PIKE-A specifically binds Akt upon EGF stimulation, and tyrosine phosphorylation is not required for this interaction (Figure 2d). 
a

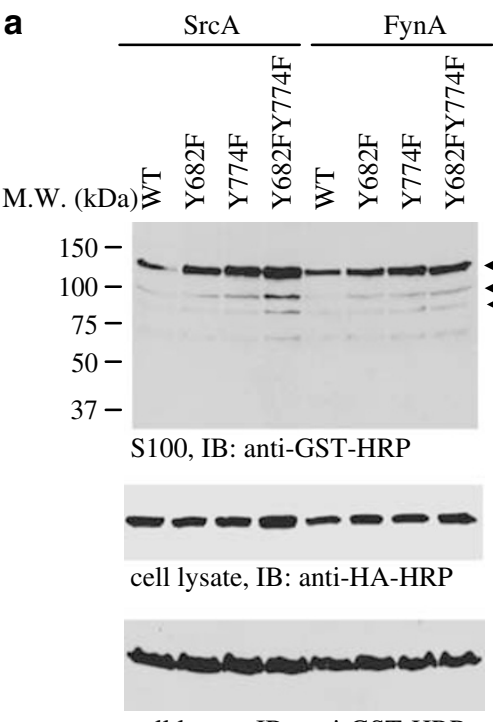

C

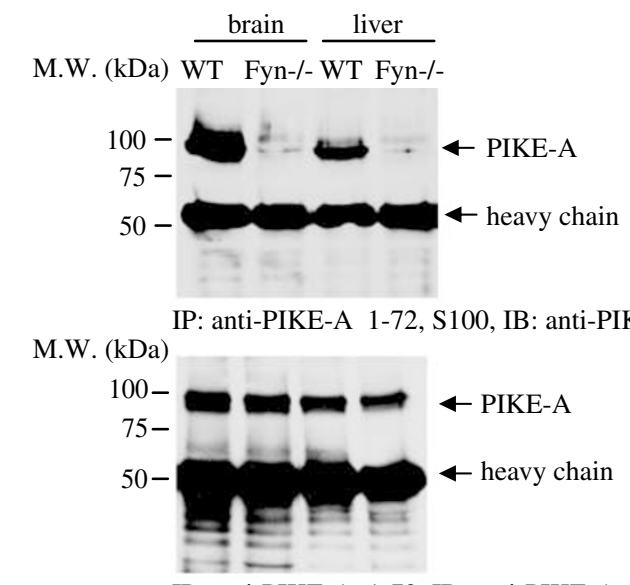

IP: anti-PIKE-A 1-72, IB: anti-PIKE-A 1-72

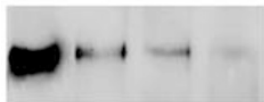

IP: anti-PIKE-A 1-72, IB: anti-PY99

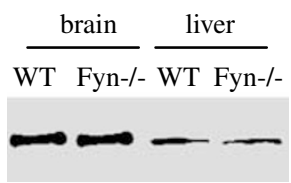

cell lysate, IB: anti-PIKE-A 1-72 b

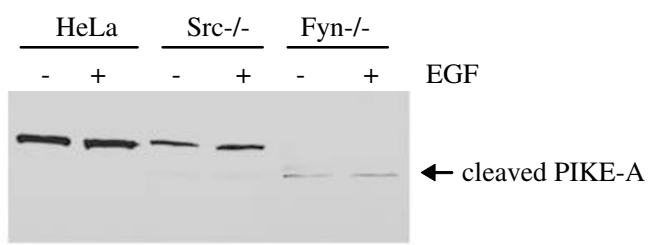

cell lysate, STS, IB: anti-PIKE-A 1-72

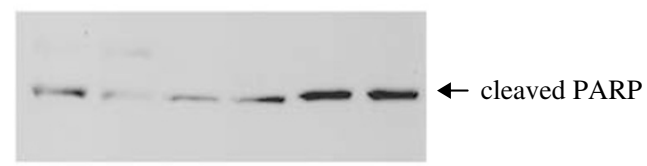

cell lysate, STS, IB: anti-PARP

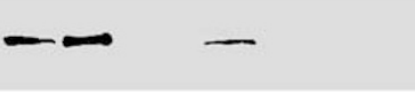

IP: anti-PIKE-A, IB: anti-PY99

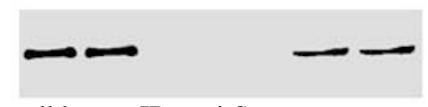

cell lysate, IB: anti-Src

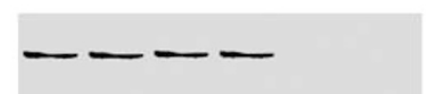

cell lysate, IB: anti-Fyn
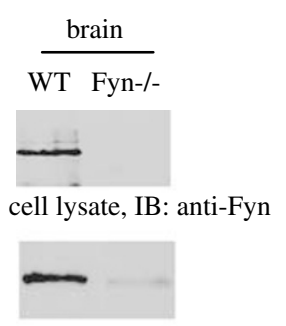

cell lysate, IB: anti-phospho-GSK-3

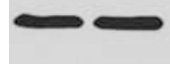

cell lysate, IB: anti-tubulin

Figure 4 Tyrosine phosphorylation prevents PIKE-A apoptotic cleavage. (a) Active fynA but not srcA protects GST-PIKE-A from apoptotic cleavage. After incubation with cell-free apoptotic solution, wild-type PIKE-A remained intact, whereas tyrosine-mutated PIKE-A was evidently cleaved. The strongest degradation occurred to the (Y682,774F) PIKE-A. (b) PIKE-A is much more vulnerable in fyn knockout cells than in src knockout cells. Most of PIKE-A remained intact in wild-type control cells. However, it was evidently cleaved in fyn-null cells with no full-length PIKE-A detected, regardless of EGF treatment; by contrast, PIKE-A apoptotic degradation was significantly diminished in src-deficient cells. As a control, PARP cleavage pattern correlated with PIKE-A cleavage (top and second panels). Tyrosine phosphorylation of PIKE-A was verified (third panel). The expression of src and fyn was confirmed in MEF cells (fourth and bottom panels). (c) PIKE-A proteolytic cleavage couples to its tyrosine phosphorylation in fyn-null mouse. PIKE-A was robustly cleaved in fyn $-I$ - sample compared to wild-type control (left top panel). Similar levels of PIKE-A were immunoprecipitated from $+I+$ and $-/-$ tissues, but more PIKE-A was pulled down from the brain than the liver (left second panel). PIKE-A was strongly phosphorylated in wild-type brain, which was markedly diminished in fyn $-/$ - brain. Its tyrosine phosphorylation in $+I+$ liver is substantially weaker than in $+I+$ brain, and almost completely abolished in $-I-$ liver (left third panel). PIKE-A was much more abundant in the brain than in the liver (left bottom panels). Fyn was selectively depleted in knockout mice (right top panel). Phosphorylation of GSK-3 was inhibited in knockout mouse (right second panel) 


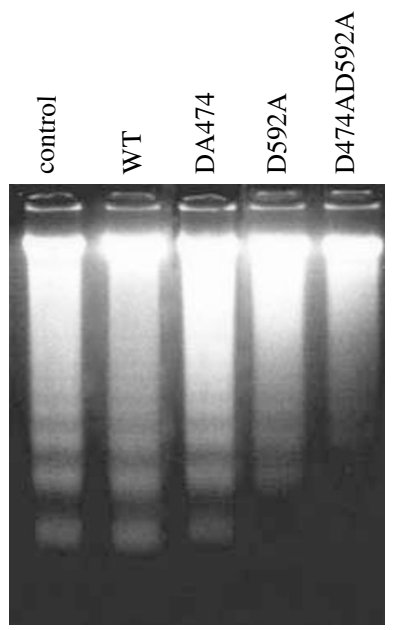

PIKE-A mutant promotes cell survival b

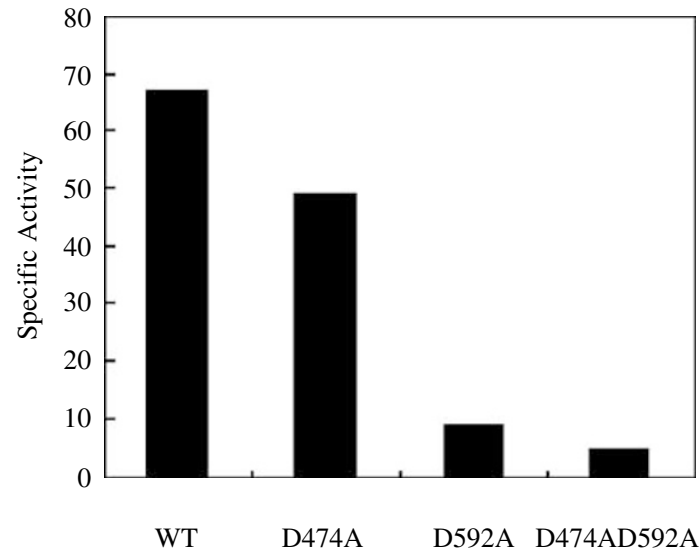

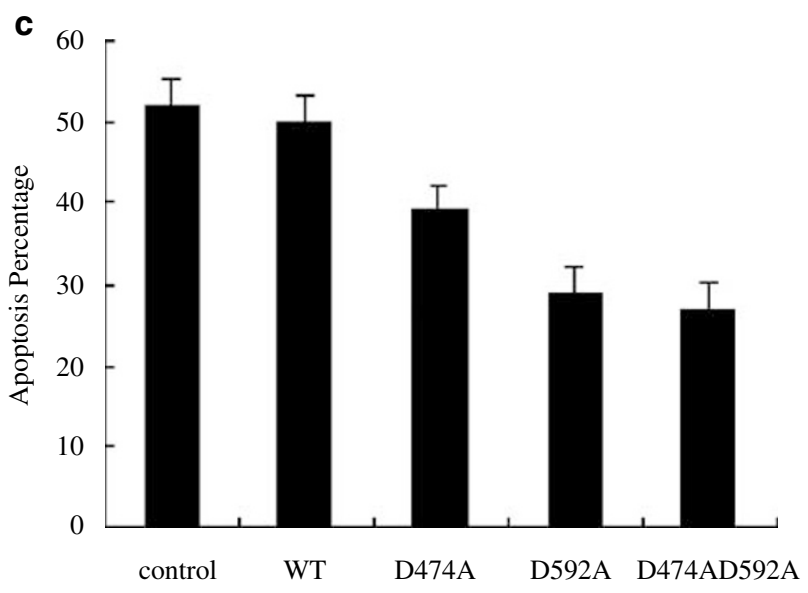

d

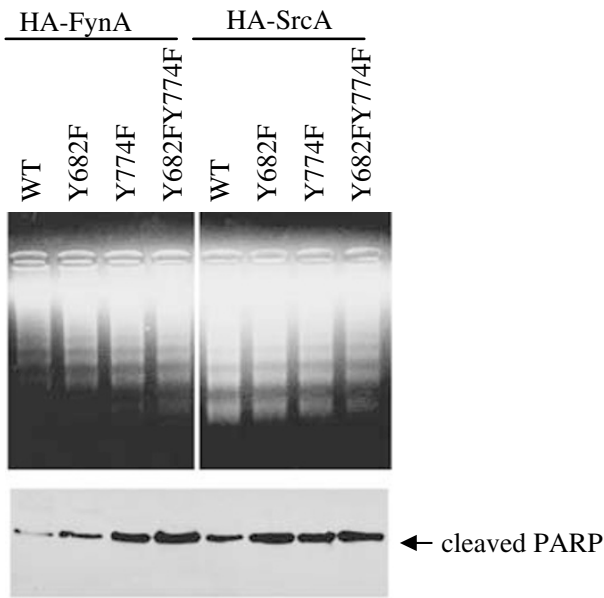

cell lysate, IB: anti-PARP

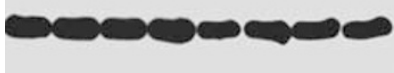

cell lysate, IB: anti-tubulin

Figure 5 Caspase-resistant PIKE-A mutants promotes cell survival. (a) Caspase-resistant PIKE-A mutant promotes cell survival. Compared with control, wild-type PIKE-A overexpression slightly decreased DNA fragmentation. The strongest inhibition occurred in PIKE-A- (D474, 592A) transfected cells, followed by D592A and D474A. (b) Caspase-3 activity correlates with DNA fragmentation pattern. (c) Quantitative analysis of the apoptotic ratio in HeLa cells. Caspase-cleavage-resistant PIKE-A mutant evidently suppressed staurosporine-induced cell death. (d) Active fyn but not src promoted PIKE-A's effect in blocking cell death. Compared with srcA, co-transfection of fynA with wild-type PIKE-A evidently diminished DNA fragmentation. By contrast, demonstrable DNA fragmentation occurred in Y682F, Y774F and Y682,774F cells (top panel). Immunoblotting analysis reveals that PARP cleavage coupled to DNA fragmentation effect (middle panel). As a control, equal amount of samples were loaded, as demonstrated by tubulin immunoblotting (bottom panel)

Our binding assay reveals that full-length PIKE-A associates with fynA through its $\mathrm{C}$-terminal ArfGAP domain. Truncation of its $\mathrm{N}$-terminal segment substantially increases the interaction between PIKE-A and fynA, although N-terminal 1-365 segments also weakly associates with fynA, suggesting that the N-terminal GTPase domain of PIKE-A and ArfGAP domain mutually mask their binding motifs for fynA (Figure 3). Conceivably, when PIKE-A is tyrosine phosphorylated, PIKEA exhibits an unfolded conformation, exposing both binding domains for fyn and Akt.

PIKE-A possesses similar structural domains as ASAP1 (ArfGAP containing $\mathrm{SH}$, ankyrin repeats and $\mathrm{PH}$ domain), which prominently binds to both FAK and pyk2 (proline-rich tyrosine $(y)$ kinase 2), a nonreceptor tyrosine kinase. ${ }^{21,22}$ Unfortunately, PIKE-A does not bind to either tyrosine kinase (data not shown). ASAP1 interacts with FAK or pyk2 through its SH3 domain, however, PIKE-A does not contain any SH3 domain. Pyk2-phosphorylating ASAP1 affects its phosphoinositides binding profiles and reduces its ArfGAP activity. ${ }^{21}$ Our previous study demonstrates that PIKE-A binds a variety of phosphatidylinositol lipids and possesses robust GTPase activity. ${ }^{23}$ It remains unknown whether fyn-mediated tyrosine phosphorylation on PIKE-A also influences the above biochemical effects. 
Earlier work shows that nonreceptor tyrosine kinases are implicated in various cellular processes including apoptosis. Some are antiapoptotic, whereas other are proapoptotic. For example, FAK plays a suppressive role in hydrogen peroxideinduced apoptosis in human glioblastoma cell line T98G. ${ }^{24}$ Overexpression of $\mathrm{v}$-src can rescue cells from apoptosis caused by cytokine deprivation or disruption of cell binding to the extracellular matrix. ${ }^{17}$ On the other hand, src family tyrosine kinase could also be proapoptotic. Both fyn and lyn but not other src family members are substrates of active caspase-3. Activation of TCR by immobilized anti-CD3 mAb triggers fyn apoptotic cleavage in T-lymphocytes. ${ }^{25}$ Activated T-lymphocytes from the fyn-deficient mice are less sensitive to killing by both anti-Fas antibody and Fas-ligand cytotoxic $T$ cells, ${ }^{26}$ indicating that fyn somehow upregulates the programmed cell death in T cells. Interestingly, overexpression in a T-cell hybridoma of a soluble form of fyn lacking the six $\mathrm{N}$ terminal amino acids thus resembling the cleaved form of fyn produced in apoptotic cells inhibits anti-CD3-induced caspase activation and apoptosis. ${ }^{27}$ Thus, cleavage of fyn and lyn upon TCR, BCR or Fas triggering could play an important role to block the apoptotic signal in a potential feedback mechanism by relocalization of the soluble forms of these kinases. ${ }^{25,27}$ This is consistent with our data that active fyn can protect PIKE-A from apoptotic cleavage and promote cell survival (Figures 4 and 5). When co-transfected with active fynA or srcA, PIKE-A is potently phosphorylated (Figure 2a). However, PIKE-A specifically suppresses apoptosis in the presence of fynA but not srcA, indicating that either PIKE-A/ fynA complex or other unknown effectors modulated by tyrosine phosphorylated PIKE-A, in addition to PIKE-A/Akt signaling cascade, contribute to inhibit apoptosis.

PIKE was initially identified as a brain-specific nuclear GTPase. Although PIKE-A isoform is ubiquitously expressed, brain tissue contains much abundant PIKE-A than liver does. Accordingly, PIKE-A tyrosine phosphorylation is much more potent in the brain than in the liver (Figure 4c). Fyn plays critical roles in many aspects of brain functions. For example, fyn is essential for myelin development in the central nervous system (CNS) and mediates learning and memory. ${ }^{13,14,28,29}$ Conceivably, fyn-mediated PIKE-A phosphorylation might regulate its biological functions in the brain. In summary, our data provide compelling evidence that active fyn is a physiological tyrosine kinase for PIKE-A. Tyrosine phosphorylation on PIKE-A by fyn protects PIKE-A from apoptotic degradation, which further enhances the antiapoptotic effect of PIKE-A. These findings establish a new mechanism how src family kinases like fyn contribute to promote cell survival.

\footnotetext{
Materials and Methods

Cells and reagents. HeLa cells and HEK293 cells were maintained in medium A (DMEM with $10 \%$ fetal bovine serum and $100 \mathrm{U}$ penicillin-streptomycin) at $37^{\circ} \mathrm{C}$ with $5 \% \mathrm{CO}_{2}$ atmosphere in a humidified incubator. Wild-type and fyn $-/-$ and src $-/-$ cells MEF cells were grown in DMEM and $10 \%$ FBS, supplemented with pyridoxine- $\mathrm{HCl}$, and $1 \mathrm{mM}$ sodium pyruvate. EGF was from Roche. Anti-PY99, caspase-3, PARP and $\alpha$-tubulin antibodies were from Santa Cruz Biotechnology Inc. Anti-Myc, HA, GST-HRP antibodies were from Sigma. Active src and fyn proteins were from Upstate Biotechnology Inc. CaspACE Assay System Colorimetric Kit was from Promega Corporation (Madison, WI, USA). All the chemicals not included above were from Sigma.
}

Cell-free apoptotic solution preparation and DNA fragmentation assay. The procedures are exactly as described. ${ }^{30}$ Briefly, the pellets of 293 cells were washed once with ice-cold PBS and resuspended in $5 \mathrm{vol}$ of buffer A, supplemented with protease inhibitors. After sitting on ice for $15 \mathrm{~min}$, the cells were broken by passing 15 times through a G22 needle. After centrifugation in a microcentrifuge for $5 \mathrm{~min}$ at $4^{\circ} \mathrm{C}$, the supernatants were further centrifuged at $10^{5} \times \mathrm{g}$ for $30 \mathrm{~min}$ in an ultracentrifuge (Beckman). The resulting supernatants were used for in vitro apoptosis assay. Cytochrome $c$ and dATP were added into S-100 extract to initiate caspase cascade. After $30 \mathrm{~min}$ incubation at $37^{\circ} \mathrm{C}$, lysates from GST-PIKE-A-transfected HEK293 cells (immunoprecipiated PIKE-A from brain and liver lysate) were introduced and incubated for $2 \mathrm{~h}$. The reaction mixture was analyzed by immunoblotting analysis with anti-GST-HRP or PIKE-A antibody, respectively. HeLa cells were transfected with various PIKE-A constructs, and treated with $1 \mu \mathrm{M}$ staurosporine for $8 \mathrm{~h}$. The DNA fragmentation assay was performed exactly as described. ${ }^{30}$

In vitro binding assays. GST fusion proteins were prepared according to the manufacturer's recommendations (Pharmacia Biotech) and the binding assay was performed as described previously. ${ }^{31}$ Twenty-four hours after transfection with $10 \mu \mathrm{g}$ of human myc-PIKE-A cDNA, a 10-cm plate of HEK293 cells was washed once in PBS, was lysed in $1 \mathrm{ml}$ lysis buffer A (50 mM Tris, pH 7.4, $40 \mathrm{mM} \mathrm{NaCl}$, $1 \mathrm{mM}$ EDTA, $0.5 \%$ Triton $\mathrm{X}-100,1.5 \mathrm{mM} \mathrm{Na}_{3} \mathrm{VO}_{4}, 50 \mathrm{mM} \mathrm{NaF}, 10 \mathrm{mM}$ sodium pyrophosphate, $10 \mathrm{mM}$ sodium $\beta$-glycerophosphate, $1 \mathrm{mM}$ phenylmethylsulfonyl flouride (PMSF), $5 \mathrm{mg} / \mathrm{ml}$ aprotinin, $1 \mathrm{mg} / \mathrm{ml}$ leupeptin, $1 \mathrm{mg} / \mathrm{ml}$ pepstatin (A)) and was centrifuged for $10 \mathrm{~min}$ at $14000 \times \mathrm{g}$ at $4^{\circ} \mathrm{C}$. From this, $500 \mu \mathrm{l}$ of supernatant were added to $50 \mu \mathrm{l}$ GST-SH3, GST-fyn (1-250) or GST-fyn (280-538) agarose, were incubated with slow rotation for $1 \mathrm{~h}$ and were washed three times with $500 \mu \mathrm{l}$ lysis buffer each time. The agarose then was resuspended in $30 \mu \mathrm{l}$ sample buffer separated by SDS-PAGE followed by immunoblot using anti-myc antibody.

Co-immunoprecipitation of HA-src family tyrosine kinases and GST-PIKE-A from transfected 293 cells. The experiments were performed as described previously. ${ }^{31}$ In brief, $10-\mathrm{cm}$ dishes of HEK293 cells were co-transfected with $5 \mu \mathrm{g}$ each of GST-PIKE-A construct and various HAtagged src family tyrosine kinases by the calcium phosphate precipitation method; the supernatant was prepared as above. After normalizing the protein concentration, $40 \mu \mathrm{l} 50 \%$ slurry glutathione beads were added to the supernatant and incubated with rotation at $4^{\circ} \mathrm{C}$ for $3 \mathrm{~h}$. The pellet was washed three times with $500 \mu \mathrm{l}$ lysis buffer each time. The agarose beads were then resuspended in $30 \mu \mathrm{l}$ sample buffer separated by SDS-PAGE followed by immunoblotting analysis using anti-HA and PY99 antibodies.

Caspase-3 activity assay. Caspase-3 activity was measured with use of the CaspACE Assay System Colorimetric Kit (Promega Corporation, Madison, WI, USA). Cells were initially seeded at a density of $1 \times 10^{6}$ in $10-\mathrm{cm}$ dishes. After staurosporine treatment for the indicated time, caspase-3 activity was measured by the cleavage of the Colorimetric substrate DEVD-pNA according to the manufacturer's instructions.

Acknowledgements. This work is supported by grant from National Institute of Health (RO1, NS045627) to K Ye.

1. Ye K, Hurt KJ, Wu FY, Fang M, Luo HR, Hong JJ et al. (2000) Pike. A nuclear gtpase that enhances PI3kinase activity and is regulated by protein 4.1N. Cell 103: 919-930.

2. Ye K, Aghdasi B, Luo HR, Moriatity JL, Wu FY, Hong JJ et al. (2002) Phospholipase $\mathrm{C}$ gamma 1 is a physiological guanine nucleotide exchange factor for the nuclear GTPase PIKE. Nature 415: 541-544.

3. Rong R, Ahn JY, Huang H, Nagata E, Kalman D, Kapp JA et al. (2003) PI3 kinase enhancer-Homer complex couples mGluRI to PI3 kinase, preventing neuronal apoptosis. Nat Neurosci 6: 1153-1161.

4. Ahn JY, Rong R, Liu X, Ye K (2004) PIKE/nuclear PI 3-kinase signaling mediates the antiapoptotic actions of NGF in the nucleus. EMBO J 23: 3995-4006.

5. Ahn JY, Hu Y, Kroll TG, Allard P, Ye K (2004) PIKE-A is amplified in human cancers and prevents apoptosis by up-regulating Akt. Proc Natl Acad Sci USA 101: 6993-6998.

6. Ahn JY, Rong R, Kroll TG, Van Meir EG, Snyder SH, Ye K (2004) PIKE (phosphatidylinositol 3-kinase enhancer)-A GTPase stimulates Akt activity and mediates cellular invasion. J Biol Chem 279: 16441-16451.

7. Tang X, Ye K (2006) PIKE tyrosine phopsphorylation regulates its apoptotic cleavage during programmed cell death. Adv Enzyme Regul (in press). 
8. Thomas SM, Brugge JS (1997) Cellular functions regulated by Src family kinases. Annu Rev Cell Dev Biol 13: 513-609.

9. Schlessinger $J(2000)$ New roles for Src kinases in control of cell survival and angiogenesis. Cell 100: 293-296.

10. Luttrell LM, Hawes BE, Biesen T, Luttrell DK, Lansing TJ, Lefkowitz RJ (1996) Role of c-Src tyrosine kinase in $\mathrm{G}$ protein-coupled receptor- and Gbetagamma subunit-mediated activation of mitogen-activated protein kinases. J Biol Chem 271: 19443-19450.

11. Kypta RM, Goldberg Y, Ulug ET, Courtneidge SA (1990) Association between the PDGF receptor and members of the src family of tyrosine kinases. Cell 62: 481-492.

12. Stein PL, Lee HM, Rich S, Soriano P (1992) pp59fyn mutant mice display differential signaling in thymocytes and peripheral T cells. Cell 70: 741-750.

13. Umemori H, Sato S, Yagi T, Aizawa S, Yamamoto T (1994) Initial events of myelination involve Fyn tyrosine kinase signalling. Nature 367: 572-576.

14. Kojima N, Wang J, Mansuy IM, Grant SGN, Mayford M, Kandel ER (1997) Rescuing impairment of long-term potentiation in fyn-deficient mice by introducing Fyn transgene. Proc Natl Acad Sci USA 94: 4761-4765.

15. Manie SN, Astier A, Haghayeghi N, Canty T, Druker B, Hirai H et al. (1997) Regulation of integrin-mediated p130(Cas) tyrosine phosphorylation in human B cells. A role for p59(Fyn) and SHP2. J Biol Chem 272: 15636-15641.

16. Beggs HE, Baragona SC, Hemperly JJ, Maness PF (1997) NCAM140 interacts with the focal adhesion kinase 125 (fak) and the SRC-related tyrosine kinase p59(fyn). J Biol Chem 272: 8310-8319.

17. McCubrey JA, Smith SR, Algate PA, DeVente JE, White MK, Steelman LS (1993) Retroviral infection can abrogate the factor-dependency of hematopoietic cells by autocrine and non-autocrine mechanisms depending on the presence of a functional viral oncogene. Oncogene 8: 2905-2915.

18. Loza-Coll MA, Perera S, Shi W, Filmus J (2005) A transient increase in the activity of Src-family kinases induced by cell detachment delays anoikis of intestinal epithelial cells. Oncogene 24: 1727-1737.

19. Seddon B, Zamoyska R (2002) TCR signals mediated by Src family kinases are essentia for the survival of naive T cells. J Immunol 169: 2997-3005.

20. Wong BR, Besser D, Kim N, Arron JR, Vologodskaia M, Hanafusa $\mathrm{H}$ et al. (1999) TRANCE a TNF family member, activates Akt/PKB through a signaling complex involving TRAF6 and c-Src. Mol Cell 4: 1041-1049.
21. Kruljac-Letunic A, Moelleken J, Kallin A, Wieland F, Blaukat A (2003) The tyrosine kinase Pyk2 regulates Arf1 activity by phosphorylation and inhibition of the Arf-GTPase-activating protein ASAP1. J Biol Chem 278: 29560-29570.

22. Liu Y, Loijens JC, Martin KH, Karginov AV, Parsons JT (2002) The association of ASAP1, an ADP ribosylation factor-GTPase activating protein, with focal adhesion kinase contributes to the process of focal adhesion assembly. Mol Biol Cell 13: 2147-2156.

23. Hu Y, Liu Z, Ye K (2005) Phosphoinositol lipids bind to phosphatidylinositol 3 (PI3)-kinase enhancer GTPase and mediate its stimulatory effect on PI3-kinase and Akt signalings. Proc Natl Acad Sci USA 102: 16853-16858.

24. Sonoda Y, Kasahara T, Yokota-Aizu E, Ueno M, Watanabe S (1997) A suppressive role of p125FAK protein tyrosine kinase in hydrogen peroxide-induced apoptosis of T98G cells. Biochem Biophys Res Commun 241: 769-774.

25. Luciano F, Ricci JE, Auberger $P$ (2001) Cleavage of Fyn and Lyn in their N-terminal unique regions during induction of apoptosis: a new mechanism for Src kinase regulation. Oncogene 20: 4935-4941.

26. Atkinson EA, Ostergaard H, Kane K, Pinkoski MJ, Caputo A, Olszowy MW et al. (1996) A physical interaction between the cell death protein Fas and the tyrosine kinase p59fynT. J Biol Chem 271: 5968-5971.

27. Ricci JE, Lang V, Luciano F, Belhacene N, Giordanengo V, Michel F et al. (2001) An absolute requirement for $\mathrm{Fyn}$ in $\mathrm{T}$ cell receptor-induced caspase activation and apoptosis. FASEB J 15: 1777-1779.

28. Nakahara J, Tan-Takeuchi K, Seiwa C, Gotoh M, Kaifu T, Ujike A et al. (2003) Signaling via immunoglobulin Fc receptors induces oligodendrocyte precursor cell differentiation. Dev Cell 4: 841-852.

29. Klein C, Kramer EM, Cardine AM, Schraven B, Brandt R, Trotter J (2002) Process outgrowth of oligodendrocytes is promoted by interaction of fyn kinase with the cytoskeletal protein tau. J Neurosci 22: 698-707.

30. Liu X, Zou H, Slaughter C, Wang X (1997) DFF, a heterodimeric protein that functions downstream of caspase-3 to trigger DNA fragmentation during apoptosis. Cell 89: 175-184.

31. Ye K, Compton DA, Lai MM, Walensky LD, Snyder SH (1999) Protein $4.1 \mathrm{~N}$ binding to nuclear mitotic apparatus protein in PC12 cells mediates the antiproliferative actions of nerve growth factor. J Neurosci 19: 10747-10756. 